\title{
Differences in oocyte development and estradiol sensitivity among mouse strains
}

\author{
Melissa E Pepling, Emily A Sundman, Nicole L Patterson, Grant W Gephardt, Leonard Medico Jr \\ and Krystal I Wilson
}

Department of Biology, Syracuse University, Syracuse, New York 13244, USA

Correspondence should be addressed to M E Pepling; Email: mepeplin@syr.edu

\begin{abstract}
Mouse oocytes develop in clusters of interconnected cells called germline cysts. Shortly after birth, the majority of cysts break apart and primordial follicles form, consisting of one oocyte surrounded by granulosa cells. Concurrently, oocyte number is reduced by two-thirds. Exposure of neonatal females to estrogenic compounds causes multiple oocyte follicles that are likely germline cysts that did not break down. Supporting this idea, estrogen disrupts cyst breakdown and may regulate normal oocyte development. Previously, the CD-1 strain was used to study cyst breakdown and oocyte survival, but it is unknown if there are differences in these processes in other mouse strains. It is also unknown if there are variations in estrogen sensitivity during oocyte development. Here, we examined neonatal oocyte development in FVB, C57BL/6, and F2 hybrid (Oct4-GFP) strains, and compared them with the CD-1 strain. We found variability in oocyte development among the four strains. We also investigated estrogen sensitivity differences, and found that C57BL/6 ovaries are more sensitive to estradiol than CD-1, FVB, or Oct4-GFP ovaries. Insight into differences in oocyte development will facilitate comparison of mice generated on different genetic backgrounds. Understanding variations in estrogen sensitivity will lead to better understanding of the risks of environmental estrogen exposure in humans.

Reproduction (2010) 139 349-357
\end{abstract}

\section{Introduction}

Establishment of functional gametes is absolutely required for successful reproduction. In mammalian females, the pool of oocytes is determined during prenatal development. Primordial germ cells (PGCs) migrate to the developing gonads from outside the growing embryo (Hirshfield 1991). In mice, upon arrival at the gonad, the PGCs undergo mitosis with incomplete cytokinesis, forming germline cysts or oocyte nests (Pepling \& Spradling 1998). After birth, two-thirds of the oocytes die by the process of programmed cell death, while the remaining oocytes separate from the cysts into single cells that become surrounded by granulosa cells to form primordial follicles (Pepling \& Spradling 2001). Thus, the processes of apoptosis and breakdown of cysts are important in establishing the pool of primordial follicles and, therefore, the fertility of the organism (Morita \& Tilly 1999).

Cyst breakdown and programmed oocyte death have been characterized in the CD-1 strain of mice, but it is not known if there are differences in these processes in other mouse strains (Pepling \& Spradling 2001). A previous study found differences in the number of total follicles at postnatal day (PND) 4 in different strains of mice (Canning et al. 2003). In contrast, at PND 42 the total number of follicles was similar in several different strains. This suggests that differences in total follicle number observed at PND 4 are somehow compensated for so that all the strains have similar numbers by PND 42. Cyst breakdown which is partially completed by PND 4 was not examined in these strains.

Many aspects of reproduction and development vary depending on the mouse strain including spermatogenesis, hormone-induced ovulation rates, and susceptibility to breast cancer (Spearow \& Barkley 1999, Spearow et al. 1999a, 1999b, Davie et al. 2007). Some strains, such as CD-1, have been selected for large litter size, and because of this other reproductive traits are coinherited (Taketo et al. 1991). Genetic variation is thought to be responsible for these differences, and thus quantitative trait loci linkage mapping can be used to begin to identify genes involved in these differences. In one study, genes controlling differences in hormoneinduced ovulation rate were mapped and compared in several strains (Spearow et al. 1999b). Three loci were identified as contributing to the observed differences. Environmental estrogens can affect spermatogenesis, and some strains such as $\mathrm{C} 57 \mathrm{Bl} / 6$ (B6) are much more sensitive to estrogenic compounds than other strains 
such as CD-1 (Spearow et al. 1999a). These differences again were attributed to genetic variation (Spearow et al. 2001).

Estrogenic compounds exert a wide variety of effects on reproductive organs including increased occurrence of multiple oocyte follicles (MOFs; Iguchi et al. 2001, Jefferson et al. 2002). In ovaries of normal adult female mice, follicles consist of one oocyte surrounded by one or more layers of granulosa cells, and follicles with more than one oocyte are rarely found (Kent 1960). However, the percentage of animals with MOFs as well as the percentage of MOFs per total follicles varies widely depending on the mouse strain (Iguchi \& Takasugi 1986, Iguchi et al. 1986). MOFs have also been reported in human ovaries (Gougeon 1981, Dandekar et al. 1988). There is evidence in mice that oocytes derived from MOFs have a reduced fertilization rate (Iguchi et al. 1990), although in humans no difference has been found (Dandekar et al. 1988). MOFs observed in mice and humans have been postulated to be remnants of oocyte clusters that did not separate and become enclosed individually in follicles during neonatal primordial follicle assembly (Gougeon 1981, Iguchi \& Takasugi 1986, Iguchi et al. 1986). Our previous work supports this idea as we found that estrogenic compounds delayed cyst breakdown and primordial follicle assembly (Jefferson et al. 2006, Chen et al. 2007). Our published model is that, normally, exposure of fetal oocytes to maternal estrogen keeps oocytes in cysts, and that at birth estrogen levels drop resulting in cyst breakdown. When neonatal oocytes are exposed to estrogens, cyst breakdown is inhibited (Chen et al. 2007). However, the idea that birth is a trigger for cyst breakdown and follicle formation is not supported by studies in other mammals where follicle formation occurs during fetal life (Gondos et al. 1971, Russe 1983).

The primary mechanism by which estrogen elicits its action is through nuclear hormone receptors, estrogen receptor $\alpha$ and $\beta$ (ER $\alpha$ and $E R \beta$, now known as ESR1 and ESR2 respectively; Britt \& Findlay 2002). During cyst breakdown, ESR1 is expressed in pregranulosa cells and ESR2 in some oocyte nuclei (Chen et al. 2009). Like estradiol $\left(E_{2}\right)$, ESR1 or ESR2 selective agonists can inhibit cyst breakdown, suggesting that estrogen can signal through either receptor to regulate cyst breakdown (Chen et al. 2009). $E_{2}$ conjugated to BSA, which can only exert effects at the membrane, was able to inhibit cyst breakdown, implying that estrogen can also function through a membrane-bound estrogen receptor to regulate cyst breakdown (Chen et al. 2009).

Steroid hormones have been implicated in the regulation of cell death in the ovary and other tissues. In the adult mammalian ovary, where most follicles undergo atresia, estrogen protects cells from dying (Billig et al. 1993). PGCs treated in culture with estrogens are stimulated to proliferate (Moe-Behrens et al. 2003). In contrast, in the nervous system, estrogen can promote cell death (Zhang et al. 2002). However, treatment of neonatal CD-1 mouse ovaries with exogenous estrogens does not alter the number of surviving oocytes (Chen et al. 2007). It is not known if there are strain differences in the effects of estrogen treatment on cyst breakdown, programmed oocyte death, and primordial follicle assembly.

Our current understanding of neonatal oocyte apoptosis and cyst breakdown is based on studies in the CD-1 strain of mice (Pepling \& Spradling 2001). To investigate strain effects, ovaries at different ages from four strains CD-1, B6, FVB, and the B6×CBA F2 hybrid (Oct4-GFP) were examined for cyst breakdown, total number of oocytes, and stage of follicle development. We found variations in neonatal oocyte number and development between strains. To investigate differences in sensitivity to estrogen, we examined the effects of estrogen treatment on cyst breakdown and primordial follicle assembly in the different strains using organ culture. B6 mice were more sensitive to $E_{2}$ treatment than the other strains examined with more oocytes at the end of the treatment period.

\section{Results}

\section{Comparison of counting techniques in neonatal ovaries}

Published counts of oocyte number in developing mouse ovaries vary widely, and it is unclear if this is due to differences between strains or methods used to assess the number of oocytes (Pepling \& Spradling 2001, Tilly 2003). To address this question, we used two different methods to count oocytes in the same strain (B6) at the same day of the development. The most common method used to count oocytes and follicles is to embed the ovary in paraffin, cut serial sections, count every fifth or tenth section, and then multiply by a correction factor to determine the total number of oocytes per ovary (Flaws et al. 2001). Another method used is an optical fraction technique where oocyte number in a fraction of the ovary is determined and used to estimate the total oocyte number (Pepling \& Spradling 2001, Myers et al. 2004). In order to compare counting techniques, we determined the number of oocytes in ovaries at PND 7 from the B6 strain using both paraffin sectioning and the optical fraction method. The numbers of oocytes counted in each ovary and the estimated total number of oocytes are shown in Table 1 . We obtained very similar numbers using both the techniques, $1313( \pm 125)$ oocytes/ovary (paraffin sectioning, $n=6$ ovaries) and $1210( \pm 164)$ oocytes/ovary (optical fraction, $n=8$ ovaries). Thus, in our hands, both methods yielded comparable results.

\section{Oocyte numbers in four different mouse strains during cyst breakdown}

We wanted to test if genetic background influences the number of oocytes a female mouse is initially endowed with or the number of oocytes lost during cyst 
Table 1 Total number of oocytes counted and estimated number of oocytes per ovary in B6 mice at postnatal day 7 using two different counting methods.

\begin{tabular}{|c|c|c|c|c|c|}
\hline \multirow[b]{2}{*}{ Ovary \# } & \multicolumn{2}{|c|}{ Paraffin sections } & \multicolumn{3}{|c|}{ Confocal analysis } \\
\hline & $\begin{array}{l}\text { Oocytes } \\
\text { counted }\end{array}$ & $\begin{array}{c}\text { Estimated total } \\
\text { number of oocytes }\end{array}$ & Ovary \# & $\begin{array}{l}\text { Oocytes } \\
\text { counted }\end{array}$ & $\begin{array}{c}\text { Estimated total } \\
\text { number of oocytes }\end{array}$ \\
\hline 1 & 177 & 1770 & 1 & 30 & 600 \\
\hline 2 & 127 & 1270 & 2 & 55 & 851 \\
\hline 3 & 112 & 1120 & 3 & 124 & 1581 \\
\hline 4 & 152 & 1520 & 4 & 103 & 1541 \\
\hline 5 & 89 & 890 & 5 & 127 & 2196 \\
\hline \multirow[t]{3}{*}{6} & 131 & 1310 & 6 & 63 & 1038 \\
\hline & & & 7 & 16 & 264 \\
\hline & & & 8 & 99 & 1636 \\
\hline Average & & 1313 & & & 1210 \\
\hline
\end{tabular}

breakdown. To determine if genetic background influences oocyte number, ovaries were collected from several different strains of mice at $16.5 \mathrm{dpc}$ through PND 4 , and oocyte number per section was determined by confocal microscopy. For all the four strains, birth occurred at $19.5 \mathrm{dpc}$. In Table 2, the number of ovaries and the total oocytes counted for each strain at each day are shown. Figure 1 shows the average oocyte number per section for four different strains at each age examined. CD-1 mice had the most oocytes at $16.5 \mathrm{dpc}$ (59.7 oocytes/section), while FVB mice had the fewest (35.7 oocytes/section). Thus, the initial number of oocytes in each strain varied, and the difference was determined to be statistically significant from CD-1 strain in the B6 and FVB strains. The rate of loss between the strains also varied with the CD-1 strain having the fastest rate of loss, and FVB having the slowest (Table 3). However, all the strains had $\sim 20$ oocytes/ section by PND 4. This represents a loss of about twothirds of the oocytes, which is similar to our previously published results (Pepling \& Spradling 2001).

\section{Cyst breakdown in different mouse strains}

In order to determine if genetic background influences the process of cyst breakdown, the ovaries collected from $16.5 \mathrm{dpc}$ through PND 4 were also analyzed for cyst breakdown. Figure 2 shows the percentage of oocytes that are no longer in cysts and are thus classified as single oocytes. At $16.5 \mathrm{dpc}$, almost all oocytes were in cysts, no single oocytes were observed in $C D-1, B 6$, or FVB strains, while two oocytes $(0.08 \%)$ were observed in the Oct4-GFP strain. Over the next 6 days, the rate of loss varied between the strains with the FVB strain having the fastest rate of cyst breakdown and CD-1 strain having the slowest (Table 3). At PND 4, more than $50 \%$ of the oocytes were single, and the FVB strain had the largest percentage of single oocytes and the percentage of single oocytes in FVB, B6, and Oct4-GFP strains was significantly higher than that in the CD-1 strain. Thus, genetically different strains begin cyst breakdown at a similar time, but proceed at different rates and by PND 4 there is variation in the amount of cyst breakdown between the strains.

We observed some oocytes that were not associated with other oocytes in cysts and appeared to have granulosa cells surrounding them at $17.5 \mathrm{dpc}, 2$ days before birth in all the four strains. In our previous work focusing on the outer cortex where the majority of the oocytes reside, we reported that in the CD- 1 strain cysts do not begin to break apart and follicles do not form until after birth (Pepling \& Spradling 2001). In the present study, we analyzed oocyte development at several different levels in the ovary. As shown in Fig. 3, oocytes close to the surface are found in large clusters (Fig. 3A), whereas deeper in the tissue some oocytes are enclosed

Table 2 Number of ovaries and total number of oocytes analyzed at each day of development from 16.5 dpc to postnatal day (PND) 4 in four mouse strains.

\begin{tabular}{|c|c|c|c|c|c|c|c|c|}
\hline \multirow[b]{2}{*}{ Age } & \multicolumn{2}{|c|}{ CD-1 } & \multicolumn{2}{|c|}{ B6 } & \multicolumn{2}{|c|}{ FVB } & \multicolumn{2}{|c|}{ Oct4 } \\
\hline & $\begin{array}{c}\text { Number of } \\
\text { ovaries }\end{array}$ & $\begin{array}{l}\text { Oocytes } \\
\text { counted }\end{array}$ & $\begin{array}{c}\text { Number of } \\
\text { ovaries }\end{array}$ & $\begin{array}{l}\text { Oocytes } \\
\text { counted }\end{array}$ & $\begin{array}{c}\text { Number of } \\
\text { ovaries }\end{array}$ & $\begin{array}{l}\text { Oocytes } \\
\text { counted }\end{array}$ & $\begin{array}{c}\text { Number of } \\
\text { ovaries }\end{array}$ & $\begin{array}{l}\text { Oocytes } \\
\text { counted }\end{array}$ \\
\hline $16.5 \mathrm{dpc}$ & 6 & 2867 & 6 & 968 & 9 & 2571 & 6 & 2479 \\
\hline $17.5 \mathrm{dpc}$ & 11 & 6090 & 11 & 3317 & 9 & 3508 & 13 & 5348 \\
\hline $18.5 \mathrm{dpc}$ & 7 & 1964 & 11 & 2706 & 8 & 2318 & 8 & 3111 \\
\hline PND 1 & 8 & 2357 & 10 & 1869 & 11 & 2557 & 8 & 1603 \\
\hline PND 2 & 9 & 1758 & 5 & 946 & 11 & 2207 & 16 & 2970 \\
\hline PND 3 & 9 & 1758 & 11 & 1790 & 7 & 1524 & 11 & 1617 \\
\hline PND 4 & 8 & 1236 & 13 & 2655 & 8 & 1274 & 11 & 2017 \\
\hline
\end{tabular}




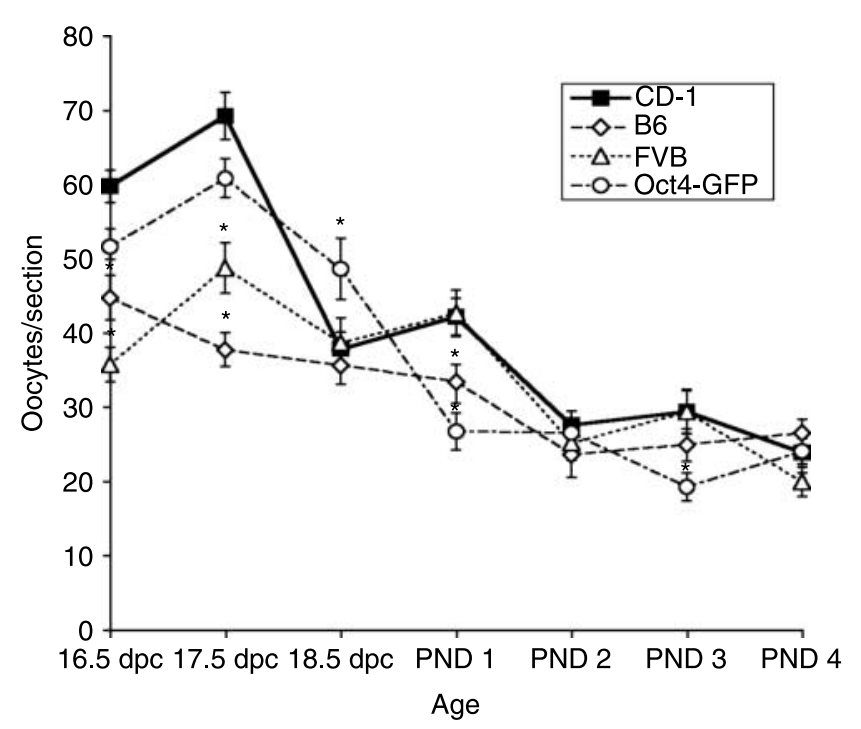

Figure 1 Number of oocytes per section at each day of development from $16.5 \mathrm{dpc}$ to PND 4 in CD-1, B6, FVB, and Oct4-GFP mouse strains. For each strain, data are presented as the mean \pm s.E.M. At least five ovaries were examined for each datapoint. *Indicates a significant difference as compared with CD-1 at the same stage of development $(P<0.05)$.

in primordial follicles (Fig. 3B). This was observed not only in the CD-1 strain but in the other strains as well. In fact, the Oct4-GFP strain had two single oocytes $(0.08 \%)$ at $16.5 \mathrm{dpc}$. Thus, primordial follicle formation began at $17.5 \mathrm{dpc}$ in three of the strains examined and at $16.5 \mathrm{dpc}$ in the Oct4-GFP strain in the medullary region of the ovary. Table 4 shows the percentage of oocytes in follicles in different levels of the ovary at $17.5 \mathrm{dpc}$. In all the four strains, the greatest percentage of oocytes in follicles was found in the deepest region of the ovary, and fewer were found in the regions toward the surface of the ovary. The B6, FVB, and Oct4 strains had a very similar distribution of follicle among the different levels of the ovary. However, the CD-1 strain had a smaller percentage in the deepest level and more oocytes in the levels near the surface.

\section{Follicle development in different mouse strains}

To determine if genetic background influences neonatal follicle development, follicle stage of the collected

Table 3 Rates of oocyte loss and cyst breakdown in neonatal mouse ovaries.

\begin{tabular}{lcc}
\hline Strain & $\begin{array}{c}\text { Oocytes lost/section } \\
\text { per day }\end{array}$ & $\begin{array}{c}\text { Percentage of } \\
\text { single oocytes/day }\end{array}$ \\
\hline CD-1 & $5.99( \pm 1.43)^{\mathrm{a}}$ & $11.66( \pm 0.01)^{\mathrm{a}}$ \\
B6 & $3.03( \pm 1.46)^{\mathrm{b}, \mathrm{c}}$ & $13.63( \pm 0.01)^{\mathrm{b}}$ \\
FVB & $2.63( \pm 1.43)^{\mathrm{c}}$ & $15.26( \pm 0.01)^{\mathrm{c}}$ \\
Oct4 & $4.61( \pm 1.40)^{\mathrm{b}}$ & $13.90( \pm 0.01)^{\mathrm{b}}$ \\
\hline
\end{tabular}

Data presented as mean \pm s.E.M. Different letter supscripts represent a significant difference between strains (univariate ANOVA, $P<0.05$ ). ovaries was assessed at $17.5 \mathrm{dpc}$ and PND 4. Figure 4 shows the percentage of follicles at each stage of development for each strain. At $17.5 \mathrm{dpc}$ in each strain, most oocytes were still in cysts and were not assembled into follicles (Fig. 4A). However, as mentioned above, a few oocytes were not associated with other oocytes and appeared to have granulosa cells surrounding them. By PND 4, though some oocytes were still not assembled into follicles, oocytes were found within primordial, primary, and even some in secondary follicles in all the strains (Fig. 4B). B6, FVB, and Oct4-GFP ovaries had significantly fewer unassembled oocytes (oocytes still in cysts) compared with CD-1 ovaries corresponding to the increased cyst breakdown in these strains. There were more oocytes contained in the primordial follicles in B6 and FVB ovaries and more primary follicles in Oct4-GFP ovaries compared with CD-1 ovaries. There were no differences in the percentage of secondary follicles in the strains examined. Thus, genetic background does influence primordial follicle assembly and follicle activation.

\section{Strain differences in estrogen sensitivity during cyst breakdown}

Some mouse strains have been reported to be much more sensitive to endocrine disruption than other strains (Spearow et al. 1999a, 1999b, 2001). We wanted to know if disruption of cyst breakdown is differentially affected by estrogen depending on the strain. To test this, we harvested ovaries from CD-1, FVB, B6, and Oct4-GFP female mice at PND 1, and grew them in culture for 7 days with $10^{-6} \mathrm{ME}_{2}$. After culture, ovarian development was analyzed. The number of oocytes/ section was significantly higher in $\mathrm{E}_{2}$-treated ovaries from all strains than in the control untreated ovaries

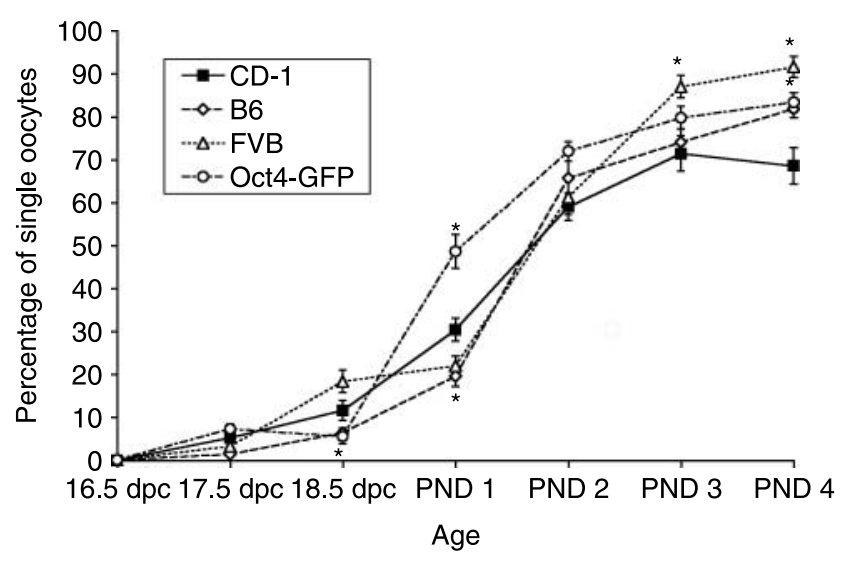

Figure 2 Comparison of oocyte cyst breakdown in several different mouse strains. The percentage of single oocytes at each day of development from 16.5 dpc to PND 4 in CD-1, B6, FVB, and Oct4-GFP mouse strains. For each strain, data are presented as the mean \pm s.E.M. At least five ovaries were examined for each datapoint. *Indicates a significant difference as compared with CD-1 at the same stage of development $(P<0.05)$. 

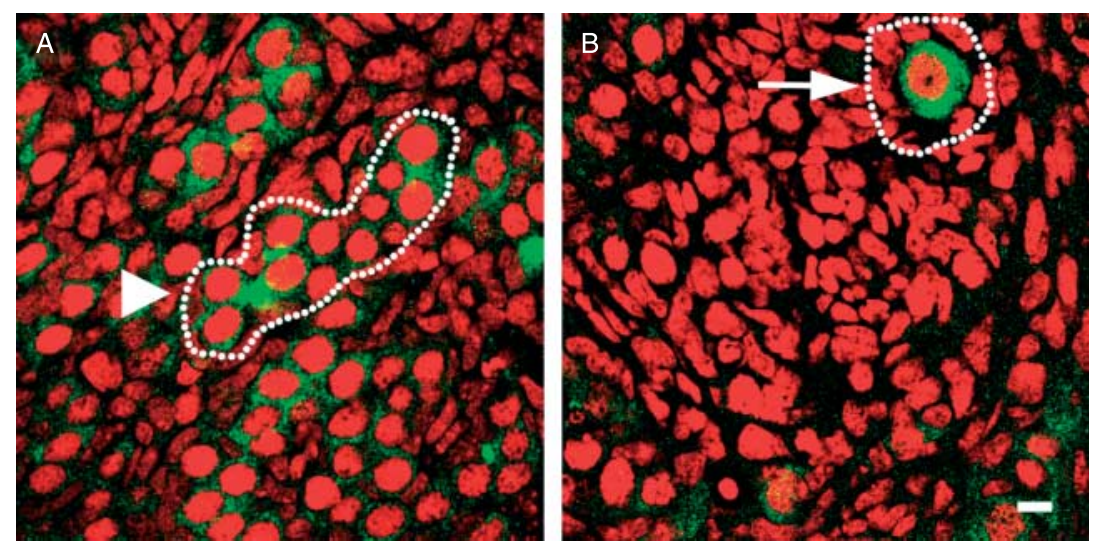

Figure 3 Representative confocal sections from $17.5 \mathrm{dpc}$ CD-1 ovaries labeled with STAT3 (green) to label oocytes and with propidium iodide (red) to visualize nuclei. (A) Confocal section near the surface of the ovary showing the arrangement of oocytes in connected cysts. The white arrowhead indicates a germ cell cyst outlined by a dotted line. (B) Confocal section near the center of the ovary showing one oocyte within a follicle (indicated by a white arrow and outlined by a dotted line). Scale bar $=10 \mu \mathrm{m}$.

(Fig. 5A). In CD-1, FVB, and Oct4-GFP ovaries, the number of oocytes was less than two times that in the controls. However, in B6 $\mathrm{E}_{2}$-treated ovaries the number of oocytes was much greater: nearly three times more than in the controls. Percentage of cyst breakdown was similar in untreated controls from all the four strains (90$100 \%$ single oocytes), and cyst breakdown was reduced in all the four strains treated with $E_{2}$ (Fig. 5B). $E_{2}$ treatment increased the number of primordial follicles in CD-1 ovaries, while in B6 ovaries there were fewer primordial follicles (Fig. 6A). The percentage of primary follicles was unaffected by $E_{2}$ in all the four strains (Fig. 6B). The percentage of secondary follicles was slightly lower in all the four strains, but was only determined to be statistically significant in the B6 strain (Fig. 6C). Thus, the B6 strain is more sensitive to $E_{2}$ than the other three strains examined with more oocytes protected from death and a delay in the transition from the primary to the secondary follicle stage.

\section{Discussion}

We found variations in oocyte number, cyst breakdown, and follicle development in all the strains examined. Surprisingly, the differences observed were not as large as we expected. One possibility is that strains not examined in this study may have greater differences. In a previous study by Canning et al. (2003) at PND 4, CD-1, FVB, and B6 mice had similar numbers of oocytes. However, the

Table 4 Numbers and percentages of oocytes enclosed in follicles at different depths of the ovary by mouse strain.

\begin{tabular}{ccccc}
\hline Depth & CD-1 & B6 & FVB & Oct4 \\
\hline Level 1 & $29(16)$ & $3(7)$ & $4(10)$ & $3(7)$ \\
Level 2 & $36(20)$ & $2(4)$ & $3(8)$ & $7(16)$ \\
Level 3 & $54(31)$ & $15(33)$ & $14(36)$ & $14(33)$ \\
Level 4 & $58(33)$ & $25(56)$ & $18(46)$ & $19(56)$
\end{tabular}

Data are presented as the number of ovaries in follicles with the percentage of follicles at each level in parentheses. Level 1 of the ovary is nearest to the outer surface of the ovary, and levels are $\sim 20 \mu \mathrm{m}$ apart with level 4 being the deepest, toward the medullary region of the ovary.
$129 /$ Sv strain had significantly more oocytes, while the AKR/J strain had significantly fewer oocytes. Cyst breakdown and oocyte survival may also be different in these strains. Strain differences have also been observed in meiotic progression (Ghafari et al. 2009).

In the studies presented here, we observed oocyte loss earlier than in our previous work which focused only on the outer cortex (Pepling \& Spradling 2001). Programmed oocyte loss and cyst breakdown begin after birth in the cortical region of the ovary, but here we observed these processes in the medullary region beginning even before birth. This is in agreement with other studies in mice and also in humans where oocyte loss has been observed during fetal development (De Pol et al. 1997, De Felici et al. 1999, McClellan et al. 2003, Ghafari et al. 2007). Follicles appear to be forming first in the innermost region of the ovary. It has been known for many years that there are regional differences in oocyte development, and that oocytes located in the inner cortex and the medullary region of the ovary enter meiosis and start to grow first (Peters 1969, Nandedkar et al. 2007). This regional pattern is set up between 13.5 and $16.5 \mathrm{dpc}$ in mice concurrent with meiotic entry (Byskov et al. 1997).

Our model of neonatal oocyte development was that before birth exposure of fetal oocytes to maternal estrogen keeps oocytes in cysts, and that at birth estrogen levels drop triggering programmed oocyte death, cyst breakdown, and primordial follicle assembly. However, in humans and in other mammals such as the cow, follicle formation does not occur around the time of birth but earlier, during gestation (Gondos et al. 1971, Russe 1983). In addition, a recent study focusing on bovine oocyte development found that estrogen produced by the fetal ovary itself inhibited the development of follicles (Yang \& Fortune 2008). In light of the results of the current study and studies of other mammals, we have modified our model. There must be a trigger of cyst breakdown that occurs before birth in the inner region of the ovary.

The number of oocytes reported in developing mouse ovaries varies depending on the laboratory and method 

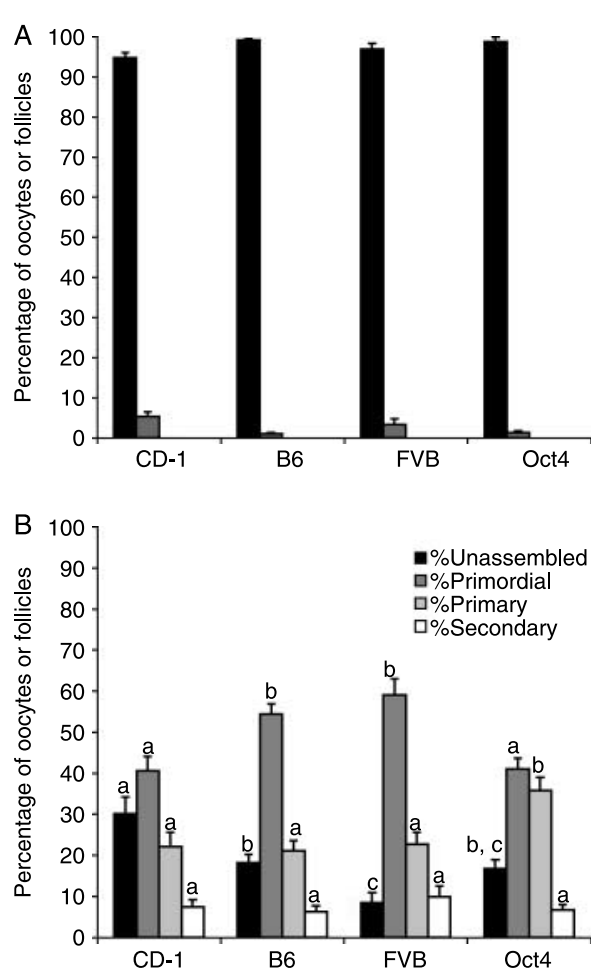

Figure 4 Analysis of primordial follicle assembly and follicle development in several different mouse strains. Percentage of unassembled oocytes, and in primordial, primary, and secondary follicles in ovaries at $17.5 \mathrm{dpc}$ (A) and PND 4 (B). Data are presented as the mean \pm s.E.M. At least five ovaries were examined for each datapoint. Different letter superscripts indicate a significant difference between strains with $P<0.05$.

used to determine oocyte number (Pepling \& Spradling 2001, Tilly 2003). We compared two different methods of counting oocytes and obtained very similar results with each method. Different numbers may be obtained when different researchers perform the same counting technique. However, the final conclusions of oocyte studies will not be affected if the same method and correction factor are applied when performing comparisons between strains.

Strain differences in sensitivity to exogenous estrogen in males have been described. In juvenile B6 male mice treated with estrogen, testes and vesicular gland weights are reduced and sperm maturation is inhibited, while CD-1 mice are resistant to these effects of estrogen treatment (Spearow et al. 1999a, 1999b, 2001). In organ culture, $E_{2}$ treatment did not affect the number of oocytes surviving in CD-1 ovaries (Chen et al. 2007). Here, we found that the B6 strain had almost three times the number of oocytes surviving after treatment with $E_{2}$, while CD-1, FVB and Oct4-GFP strains had less than two times the number of oocytes and thus $\mathrm{B} 6$ mice are more sensitive to exogenous $E_{2}$ than the CD-1, FVB and Oct4GFP strains. In our previous studies, we found that treatment of CD-1 neonates with genistein by injection protected more oocytes from dying, while genistein treatment of neonatal ovaries in culture did not result in more oocytes (Jefferson et al. 2006, Chen et al. 2007). These differences may be because the ovary is being exposed to the hormone in different manners. However, unlike genistein, neither $\mathrm{E}_{2}$ treatment in vivo nor $\mathrm{E}_{2}$ treatment in organ culture had an effect on the number of oocytes (Chen et al. 2007). It could be that factors other than $\mathrm{E}_{2}$ are more important in regulating neonatal oocyte death, and that estrogen is specific to cyst breakdown. It may also be that by PND 1 when we begin the treatment, the determination of an oocyte to die has already occurred and earlier treatments might rescue more of the oocytes from death. Interestingly, treatment with estrogenic compounds earlier from 11.5 to $16.5 \mathrm{dpc}$ in mice does not affect follicle formation (Sonne-Hansen et al. 2003).

$\mathrm{E}_{2}$ treatment also affected follicle development in all the four strains examined. We found that there were fewer secondary follicles in all strains, but the difference was only statistically significant in the B6 strain suggesting that $E_{2}$ can delay the development of follicles from the primary to the secondary stage. Studies of the effects of $E_{2}$ treatment on fetal bovine ovaries also found similar effects on follicle development (Yang \& Fortune 2008).

Insight into the strain differences of oocyte survival, cyst breakdown, and primordial follicle formation and sensitivity to endocrine disruptors will lead to a better
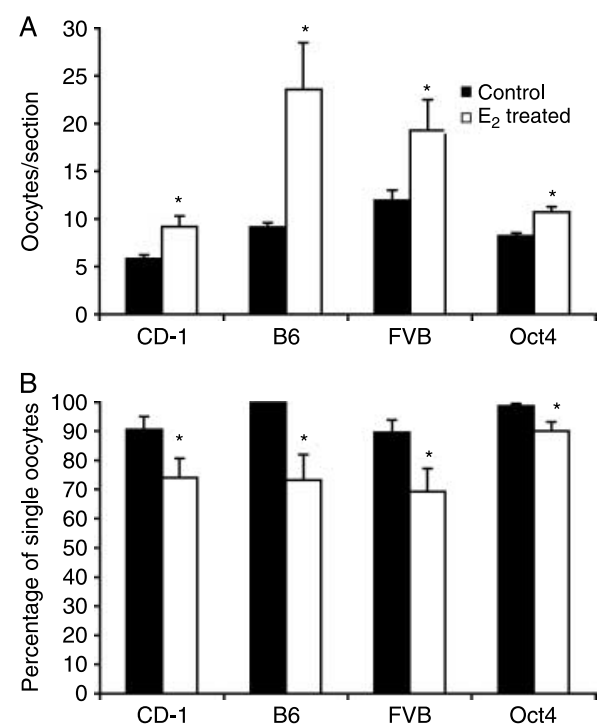

Figure 5 Changes in oocyte number and cyst breakdown in different mouse strains in response to estradiol. Number of oocytes per confocal section (A) and percentage of single oocytes (B) in control and treated newborn ovaries after 7 days of culture with no hormone added or with $10^{-6} \mathrm{M}$ estradiol. Data are presented as the mean \pm S.E.M. ${ }^{*}$ Indicates a significant difference between control and treated ovaries (ANOVA, $P<0.05)$. $N=3-5$ ovaries per group. 


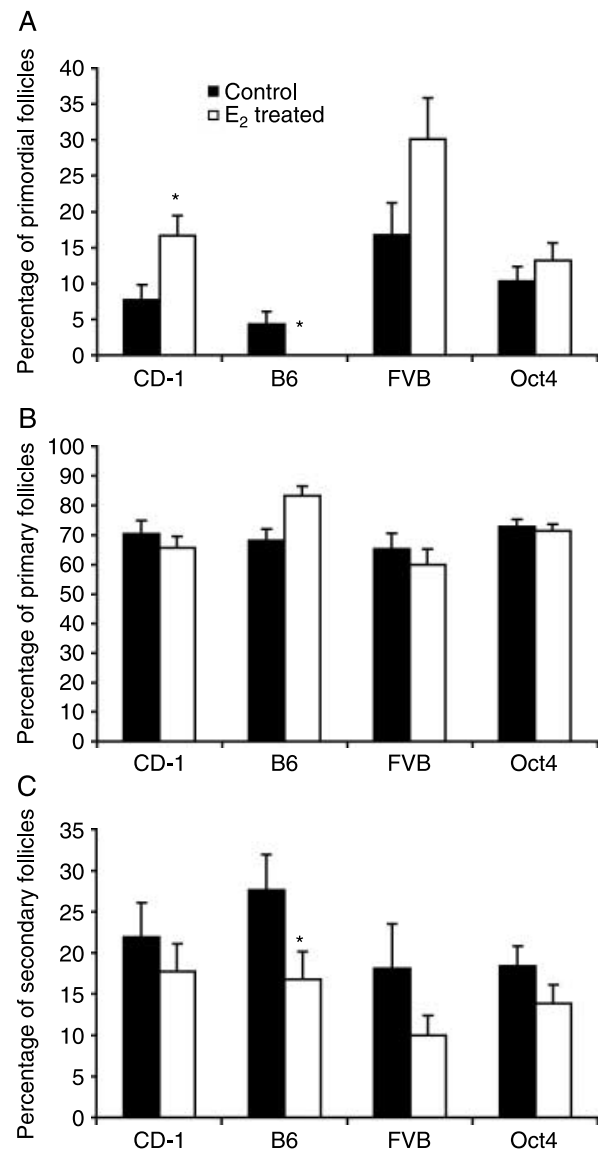

Figure 6 Follicle development in different mouse strains treated with estradiol. Percentage of primordial (A), primary (B), and secondary follicles $(\mathrm{C})$ relative to the total number of follicles in control and treated newborn ovaries after 7 days of culture with no hormone added or with $10^{-6} \mathrm{M}$ estradiol. Data are presented as the mean \pm S.E.M. *Indicates a significant difference between control and treated ovaries (ANOVA, $P<0.05)$. $N=3-5$ ovaries per group.

understanding of the forces that influence germ cell population and fertility. Strain differences in neonatal oocyte development are important to keep in mind when performing mutant analyses and examining exogenous hormone effects.

\section{Materials and Methods}

\section{Animals}

Ovaries from neonatal female mice were obtained from the CD-1 strain (Charles River Laboratories, Wilmington, MA, USA), B6 strain (The Jackson Laboratory, Bar Harbor, ME, USA), FVB strain (generously provided by Dr Jodi Flaws, University of Maryland), and a B6×CBA F2 hybrid strain expressing GFP in oocytes under the control of the Oct4 promoter, TgOG2 (Oct4-GFP; The Jackson Laboratory; Szabo et al. 2002). The presence of a vaginal plug was used to determine that mating occurred the night before, and was designated $0.5 \mathrm{dpc}$. Plugged female mice were isolated from males. The day of birth of the pups was designated PND 1 and was 19 days after a plug was found. All animal experimentation was reviewed and approved by the Syracuse University Institutional Animal Care and Use Committee.

\section{Paraffin embedding}

PND 7 B6 ovaries for embedding and sectioning were harvested and fixed in 10\% buffered formalin (Fisher Scientific, Pittsburgh, PA, USA). Both ovaries from each neonate were used. Fixed ovaries were placed in an embedding cassette and washed in running water, $70 \%$ ethanol, $95 \%$ ethanol, and $100 \%$ ethanol. The tissues were immersed in clearing oil, dehydrated in xylene, and soaked in paraffin. Base molds were heated, and a small amount of melted paraffin was added. The tissue was embedded in paraffin and sectioned at a thickness of $8 \mu \mathrm{m}$.

\section{Hematoxylin and eosin staining}

Sections were rehydrated in xylene, 100\% ethanol, 95\% ethanol, $70 \%$ ethanol, $50 \%$ ethanol, and water, and were then stained in Mayer's hematoxylin (Richard-Allan Scientific, Kalamazoo, MI, USA). Sections were placed in Clarifier 1 (Richard-Allan Scientific) and were then placed in Bluing Reagent (Richard-Allan Scientific), cleared in 70\% ethanol, and counterstained with Eosin (Richard-Allan Scientific). The sections were dehydrated in $100 \%$ ethanol followed by xylene and then mounted with Permount (Fisher Scientific, Waltham, MA, USA).

The number of oocytes was calculated from paraffinembedded, hematoxylin, and eosin PND 7 B6 ovaries. In every tenth section, the numbers of oocytes were determined and then multiplied by 10 to estimate the total number of oocytes in the ovary. Only oocytes containing a visible nucleus were counted to avoid double counting.

\section{Immunohistochemistry}

Whole CD-1, B6, FVB, and Oct4-GFP ovaries were dissected in PBS and fixed in 5\% EM grade paraformaldehyde (Ted Pella, Inc., Redding, CA, USA) in PBS overnight at $4{ }^{\circ} \mathrm{C}$ followed by several washes in $0.1 \%$ Triton X-100 in PBS, and were then incubated with $5 \%$ BSA in $0.1 \%$ Triton X-100 in PBS to block nonspecific binding. Both ovaries of each neonate were used except in a few cases where one of the ovaries was not well stained. Whole ovaries were immunostained as described previously (Murphy et al. 2005). The STAT3 (C20) antibody (Santa Cruz Biotechnology, La Jolla, CA, USA), a specific marker for germ cells, was used at a dilution of 1:500 (Murphy et al. 2005). The secondary antibody antirabbit Alexa 488 (Molecular Probes, now part of Invitrogen) was used at a dilution of 1:200. Propidium iodide (Molecular Probes, now part of Invitrogen) was used to label the nuclei. Nuclear morphology was used to confirm the identification of the germ cells. Oct4-GFP ovaries were not labeled with STAT3 antibody, and instead, GFP expression was used to identify the oocytes. 


\section{Confocal microscopy}

The distribution of germ cells within the ovary is asymmetric, including along the dorsal-ventral axis. As such, after mounting the stained ovaries, they were examined and flipped appropriately so that all the dorsal sides faced the same direction. Using a LSM Pascal 5 confocal microscope (Carl Zeiss Microlmaging, Inc., Thornwood, NY, USA), a low magnification image of each ovary was taken with the $10 \times$ objective in order to capture a whole ovary image. Then, all the subsequent images were taken with a $63 \times$ water objective. Four single images starting at the dorsal surface of the ovary $\sim 20 \mu \mathrm{m}$ apart were taken to completely sample through each ovary. Each set of four images, spaced out along the focal path without moving the stage, is termed a core. Two such cores were taken from every ovary. In addition, a z-stack of 11 images ( $1 \mu \mathrm{m}$ apart) was taken centered around each of the four original single images.

\section{Optical fraction method for calculating the number of oocytes per ovary}

The optical fraction method was used to calculate the total number of oocytes per ovary from whole ovaries labeled with Stat3 for the B6 strain at PND 7 as described previously (Pepling \& Spradling 2001). Both ovaries of each neonate were used. The height, width, and depth of each ovary were measured and used to calculate the volume of the ovary. Then, the fraction of the ovary volume represented by the counted core was determined using the area of the ovary examined and taking into account the average diameter of the oocytes. Using this information, the number of oocytes for the whole ovary was extrapolated from the number in the counted core. In all the subsequent experiments examining strain differences in oocyte development and $E_{2}$ sensitivity, we determined the number of oocytes in a defined region of the ovary using confocal microscopy (the core) and did not extrapolate to the whole ovary since we were comparing similar regions between samples.

\section{Analysis of oocyte cyst breakdown, primordial follicle assembly, follicle development, and oocyte number}

Images were analyzed using the LSM image browser program (Carl Zeiss Microlmaging, Inc). Ovaries were examined for the percentage of single oocytes relative to the total number of oocytes to assess oocyte cyst breakdown (Pepling \& Spradling 2001, Jefferson et al. 2006). The number of individual oocytes relative to the number of oocytes in cysts was determined by examining the two core samples taken from each ovary. The z-stack of sections was used to determine if oocytes in the center section were associated with oocyte cysts above or below the plane of focus. For primordial follicle assembly, oocytes were considered unassembled if STAT3 antibody labeling showed that the oocytes were associated. For primordial follicle development, the number of each type of follicle per region was determined. Follicles were classified as follows: primordial (oocyte surrounded by several flattened granulosa cells), primary (oocyte surrounded by one layer of cuboidal granulosa cells), or secondary (oocyte surrounded by more than one layer of granulosa cells). The number of oocytes per section was determined by counting the number of oocytes in the sections that were collected for the analysis of cyst breakdown.

\section{In vitro ovary organ culture}

Ovaries were collected at PND 1 and placed into culture. Ovaries were cultured in drops of media on $0.4 \mu \mathrm{m}$ floating filters (Millicell-CM, Millipore Corp., Bedford, MA, USA) in $0.4 \mathrm{ml}$ DMEM-Ham's F-12 media supplemented with penicillin-streptomycin, $5 \times$ ITS-X (Life Technologies, Inc.), $0.1 \%$ BSA, $0.1 \%$ albumax, and $0.05 \mathrm{mg} / \mathrm{ml}$ L-ascorbic acid in 4-well culture plates. $\mathrm{E}_{2}$ (Sigma Chemical Company) was dissolved in DMSO at a concentration of $0.1 \mathrm{M}$ and was then added to the culture media to achieve the desired final concentration of $10^{-6} \mathrm{M}$. This concentration of $\mathrm{E}_{2}$ was chosen because it had a strong effect on CD-1 ovaries in our previous studies (Chen et al. 2007). This concentration is higher than expected with physiological conditions because we were testing response to exogenous $E_{2}$ exposure. DMSO was added to the media at the same percentage as in hormone treatment $(<0.1 \%)$ to serve as a vehicle control. Ovaries were exposed daily to $E_{2}$ for 7 days. All ovaries were processed for whole mount immunohistochemistry.

\section{Statistical analysis and calculations}

One-way ANOVA was conducted to analyze strain differences in oocyte number, percentage of single oocytes, and follicle development. PROC GLM of SAS 9.1 (SAS Institute Inc., Cary, $\mathrm{NC}$, USA) was used to calculate the least-squares means and test-specific hypotheses for effects. $P<0.05$ was considered significant. JMP 5.0.1.2 (SAS Institute) was used to analyze $E_{2}$ treatment effects on oocyte number, percentage of single oocytes, and follicle development in organ culture. Again, $P<0.05$ was considered significant. Univariate ANOVA using the least significant difference post hoc test was used to analyze strain differences in the rate of oocyte loss and cyst breakdown.

\section{Declaration of interest}

The authors declare that there is no conflict of interest that could be perceived as prejudicing the impartiality of the research reported.

\section{Funding}

This work was supported by National Science Foundation grant IOB-0613895 (to M E Pepling).

\section{Acknowledgements}

We thank Brian Calvi and Kelsey Breen for helpful comments on the manuscript; Ying Chen, Nancy Hughes, and Kari Segraves for help with statistics; and Jodi Flaws for the FVB mouse strain. 


\section{References}

Billig H, Furuta I \& Hsueh AJ 1993 Estrogens inhibit and androgens enhance ovarian granulosa cell apoptosis. Endocrinology 133 2204-2212.

Britt KL \& Findlay JK 2002 Estrogen actions in the ovary revisited. Journal of Endocrinology 175 269-276.

Byskov AG, Guoliang X \& Andersen CY 1997 The cortex-medulla oocyte growth pattern is organized during fetal life: an in vitro study of the mouse ovary. Molecular Human Reproduction 3 795-800.

Canning J, Takai Y \& Tilly JL 2003 Evidence for genetic modifiers of ovarian follicular endowment and development from studies of five inbred mouse strains. Endocrinology 144 9-12.

Chen Y, Jefferson WN, Newbold RR, Padilla-Banks E \& Pepling ME 2007 Estradiol, progesterone, and genistein inhibit oocyte nest breakdown and primordial follicle assembly in the neonatal mouse ovary in vitro and in vivo. Endocrinology 148 3580-3590.

Chen Y, Breen K \& Pepling M 2009 Estrogen can signal through multiple pathways to regulate oocyte cyst breakdown and primordial follicle assembly in the neonatal mouse ovary. Journal of Endocrinology 202 407-417.

Dandekar PV, Martin MC \& Glass RH 1988 Polyovular follicles associated with human in vitro fertilization. Fertility and Sterility 49 483-486.

Davie SA, Maglione JE, Manner CK, Young D, Cardiff RD, MacLeod CL \& Ellies LG 2007 Effects of FVB/NJ and C57BI/6J strain backgrounds on mammary tumor phenotype in inducible nitric oxide synthase deficient mice. Transgenic Research 16 193-201.

De Felici M, Di Carlo A, Pesce M, Iona S, Farrace MG \& Piacentini M 1999 $\mathrm{Bcl}-2$ and Bax regulation of apoptosis in germ cells during prenatal oogenesis in the mouse embryo. Cell Death and Differentiation 6 908-915.

De Pol A, Vaccina F, Forabosco A, Cavazzuti E \& Marzona L 1997 Apoptosis of germ cells during human prenatal oogenesis. Human Reproduction 12 2235-2241.

Flaws JA, Hirshfield AN, Hewitt JA, Babus JK \& Furth PA 2001 Effect of $\mathrm{Bcl}-2$ on the primordial follicle endowment in the mouse ovary. Biology of Reproduction 64 1153-1159.

Ghafari F, Gutierrez CG \& Hartshorne GM 2007 Apoptosis in mouse fetal and neonatal oocytes during meiotic prophase one. BMC Developmental Biology 787

Ghafari F, Pelengaris S, Walters E \& Hartshorne GM 2009 Influence of p53 and genetic background on prenatal oogenesis and oocyte attrition in mice. Human Reproduction 24 1460-1472.

Gondos B, Bhiraleus P \& Hobel CJ 1971 Ultrastructural observations on germ cells in human fetal ovaries. American Journal of Obstetrics and Gynecology 110 644-652.

Gougeon A 1981 Frequent occurrence of multiovular follicles and multinuclear oocytes in the adult human ovary. Fertility and Sterility 35 417-422.

Hirshfield AN 1991 Development of follicles in the mammalian ovary. International Review of Cytology 124 43-101.

Iguchi T \& Takasugi N 1986 Polyovular follicles in the ovary of immature mice exposed prenatally to diethylstilbestrol. Anatomica Embryologica 175 53-55.

Iguchi T, Takasugi N, Bern HA \& Mills KT 1986 Frequent occurrence of polyovular follicles in ovaries of mice exposed neonatally to diethylstilbestrol. Teratology 34 29-35.

Iguchi T, Fukazawa Y, Uesugi Y \& Taksugi N 1990 Polyovular follicles in mouse ovaries exposed neonatally to diethylstibestrol in vivo and in vitro. Biology of Reproduction 43 478-484.

Iguchi T, Watanabe H \& Katsu Y 2001 Developmental effects of estrogenic agents on mice, fish, and frogs: a mini-review. Hormones and Behavior 40 248-251.

Jefferson WN, Couse JF, Padilla-Banks E, Korach KS \& Newbold RR 2002 Neonatal exposure to genistein induces estrogen receptor (ER)alpha expression and multioocyte follicles in the maturing mouse ovary: evidence for ERbeta-mediated and nonestrogenic actions. Biology of Reproduction 67 1285-1296.

Jefferson W, Newbold R, Padilla-Banks E \& Pepling M 2006 Neonatal genistein treatment alters ovarian differentiation in the mouse: inhibition of oocyte nest breakdown and increased oocyte survival. Biology of Reproduction 74 161-168.
Kent HA 1960 Polyovular follicles and multinucleate ova in the ovaries of young mice. Anatomical Record 137 521-524.

McClellan KA, Gosden R \& Taketo T 2003 Continuous loss of oocytes throughout meiotic prophase in the normal mouse ovary. Developmental Biology 258 334-348.

Moe-Behrens GH, Klinger FG, Eskild W, Grotmol T, Haugen TB \& De Felici M 2003 Akt/PTEN signaling mediates estrogen-dependent proliferation of primordial germ cells in vitro. Molecular Endocinology 17 2630-2638.

Morita Y \& Tilly JL 1999 Oocyte apoptosis: like sand through an hourglass. Developmental Biology 213 1-17.

Murphy K, Carvajal L, Medico L \& Pepling ME 2005 Expression of Stat3 in germ cells of developing and adult mouse ovaries and testes. Gene Expression Patterns 5 475-482.

Myers M, Britt KL, Wreford NGM, Ebling FJP \& Kerr JB 2004 Methods for quantifying follicular numbers within the mouse ovary. Reproduction 127 569-580.

Nandedkar T, Dharma S, Modi D \& Dsouza S 2007 Differential gene expression in transition of primordial to preantral follicles in mouse ovary. Society of Reproduction and Fertility Supplement 63 57-67.

Pepling ME \& Spradling AC 1998 Female mouse germ cells form synchronously dividing cysts. Development 125 3323-3328.

Pepling ME \& Spradling AC 2001 The mouse ovary contains germ cell cysts that undergo programmed breakdown to form follicles. Developmental Biology 234 339-351.

Peters H 1969 The effect of radiation in early life of the morphology and reproductive function of the mouse ovary. In Advances in Reproductive Physiology, pp 149-185. Ed. A McLaren. Edinburgh: Logos Press Limited.

Russe I 1983 Oogenesis in cattle and sheep. Bibliotheca Anatomica 24 77-92.

Sonne-Hansen K, Nielsen M \& Byskov AG 2003 Oocyte number in newborn mice after prenatal octylphenol exposure. Reproductive Toxicology 17 59-66.

Spearow JL \& Barkley M 1999 Genetic control of hormone-induced ovulation rate in mice. Biology of Reproduction 61 851-856.

Spearow JL, Doemeny P, Sera R, Leffler R \& Barkley M 1999a Genetic variation in susceptibility to endocrine disruption by estrogen in mice. Science 285 1259-1261.

Spearow JL, Nutson PA, Mailliard WS, Porter M \& Barkley M 1999b Mapping genes that control hormone-induced ovulation rate in mice. Biology of Reproduction 61 857-872.

Spearow JL, O'Henley P, Doemeny P, Sera R, Leffler R, Sofos T \& Barkley M 2001 Genetic variation in physiological sensitivity to estrogen in mice. Acta Pathologica, Microbiologica, et Immunologica Scandinavica 109 356-364.

Szabo PE, Hubner K, Scholer H \& Mann JR 2002 Allele-specific expression of imprinted genes in mouse migratory primordial germ cells. Mechanisms of Development 115 157-160.

Taketo M, Schroeder AC, Mobraaten LE, Gunning KB, Hanten G, Fox RR, Roderick TH, Stewart CL, Lilly F, Hansen CT et al. $1991 \mathrm{FVB} / \mathrm{N}$ : an inbred mouse strain preferable for transgenic analyses. PNAS $\mathbf{8 8}$ 2065-2069.

Tilly JL 2003 Ovarian follicle counts - not as simple as 1, 2, 3. Reproductive Biology and Endocrinology 111

Yang MY \& Fortune JE 2008 The capacity of primordial follicles in fetal bovine ovaries to initiate growth in vitro develops during mid-gestation and is associated with meiotic arrest of oocytes. Biology of Reproduction 78 1153-1161.

Zhang L, Li BS, Zhao W, Chang YH, Ma W, Dragan M, Barker JL, Hu Q \& Rubinow DR 2002 Sex-related differences in MAPKs activation in rat astrocytes: effects of estrogen on cell death. Molecular Brain Research $1031-11$.

Received 20 September 2009

First decision 7 October 2009

Accepted 20 October 2009 